\title{
We Asked the Experts: Global Surgery-Seeing Beyond the Silo
}

\author{
Grace Umutesi $^{1}$ (1) $\cdot$ Justine Davies $^{2,3} \cdot$ Bethany L. Hedt-Gauthier $^{4,5}$
}

Accepted: 9 August 2020/Published online: 18 August 2020

(C) Société Internationale de Chirurgie 2020

The COVID-19 pandemic requires comprehensive health systems response, with $14 \%$ of infected people developing severe sickness leading to hospitalization and 5\% admitted to an intensive care unit [1]. The need for oxygen and intensive care means that perhaps for the first time, surgery and anesthesia find themselves playing a central role in a global health emergency; but is global surgery integrated enough to the wider global health community to have an impact?

Global surgery programs are relatively new and, with some recent exceptions, placed within institutions in highincome countries (HICs). Inspired by the 2015 Lancet Commission on Global Surgery [2], with "access to safe and affordable surgery" as their core mission, these programs are largely populated with surgeons, anesthesiologists and obstetricians focused on building the surgical workforce and related ecosystem in low- and middle-income countries (LMICs). Reflecting the expertise of these groups, interventions often center on the operating room, including clinical trials of surgery delivery. However, improving quality of surgery per se and expanding the

Justine Davies

J.Davies.6@bham.ac.uk

1 Partners In Health/Inshuti Mu Buzima, Rwinkwavu, Rwanda

2 Institute for Applied Research, University of Birmingham, Birmingham B15 2TT, UK

3 Department of Global Health, Centre for Global Surgery, Stellenbosch University, Stellenbosch, South Africa

4 Department of Global Health and Social Medicine, Harvard Medical School, Boston, MA, USA

5 Program in Global Surgery and Social Change, Harvard Medical School, Boston, MA, USA surgical workforce alone cannot fully address the needs of the 5 billion people lacking access to surgical care [2].

This point is well illustrated by considering the threedelay model of access to quality care. Barriers that patients experience start at the delay of "seeking care," loosely, when patients recognize that they have a disease that can be successfully treated without fear that treatment will cause them financial hardship. Overcoming hurdles in seeking care requires population health education and the provision of healthcare services that patients know they can afford and that instill patients trust. The second delay of "reaching care" requires that affordable transport is available to take patients to a facility that can treat them in a timely manner. Solutions include investment in transport solutions-both community transport options and ambulances - and the availability of easily reachable essential services. The third delay is "receiving quality care" once at a healthcare facility. This is where surgeons have most input, in delivering quality clinical surgical outcomes. However, to achieve good perioperative outcomes, health systems must address the entire patient clinical experience. This includes a safe physical environment for surgery and recovery; the availability of equipment and commodities to conduct surgery; sufficient numbers of qualified staff from a variety of disciplines ranging from custodial staff to nurses, pharmacists to physiotherapists, and laboratory technicians to biomedical engineers; and interventions to ensure services are affordable to all and availed equitably.

Importantly, a fourth delay has recently been added to the model-retaining surgical patients in care after discharge until they have rehabilitated to optimal function [3]. This requires linked referral systems, the availability of affordable ongoing specialized care (e.g., physiotherapy), and patients recognizing the necessity of their ongoing care. These delays have important consequences for 
patients. For trauma, the first two delays, which remain invisible to most surgeons, are associated with $40 \%$ of avoidable deaths [4]. Within facilities, mortality from the congenital abnormality, gastroschisis, in HIC is $<5 \%$ compared to approaching $100 \%$ in many LMICs; much of this difference is driven by lack of parenteral nutrition and neonatal intensive care capacity, as well as delays in seeking and reaching care in LMICs [5]. Although neonates with this condition are traditionally cared for by surgeons, focusing on surgery alone fails to improve outcomes for this condition. In our own work on cesarean deliveries in rural Rwanda, we observed that financial barriers and the physical burden of traveling for postoperative care limit comprehensive follow-up and are linked to increased risk of surgical site infections.

Solutions ensuring that patients complete this pathway with optimal outcomes are multifaceted, and some solutions are more directly under a surgeon's control than others. For example, being considerate to patients' needs requires a change of attitude among care providers. Advocating for Universal Health Coverage for surgery requires a massive commitment from all players. However, we argue that this massive commitment is absolutely necessary. If we are truly to improve access to quality surgery care in LMICs, all surgeons need to see outside of the hospital and act as advocates for all of their patients' needs, not accepting barriers as normal occurrences, but taking note of them, showing resultant adverse effects, and using this knowledge to advocate for change. A deep understanding of the context in which care is delivered and the full patient experience is required for this, and as such, the field of global surgery must expand to embrace a broader approach.

Treating global surgery as a health systems issue and, engaging the entire system to improve access to safe and affordable surgical care, will have huge benefits for other non-surgical conditions. Previous work assessing anesthesia capacity in 22 LMICs highlighted that over a third of facilities had no access to oxygen and no anesthesia machine, but this finding only provoked realization of the need for action during a global pandemic [6]. The misconception that surgical care is "luxury" with high price tags, therefore unobtainable in LMICs, must be replaced with the notion that high-quality surgical care is the cornerstone of effective health systems. Just as we call for the global surgery community to embrace a health systems approach, this shift requires that all who are interested in improving health globally understand more about surgery, and see colleagues from the surgical community who want to collaborate as beneficial to their work, not a threat.

As three health systems researchers operating in the space of global surgery, we see great opportunity to improve actions toward increased access to safe surgery in LMICs, specifically recognizing that global surgery requires a whole health system approach. In forgetting this, poor coordination of service delivery will remain a challenge. Keeping this recognition at the fore of any planning efforts will foster strong partnerships and an efficient use of resources, not just for the 5 billion who lack access to surgical care, but for all needing comprehensive, highquality services across all health conditions around the world.

\section{Compliance with Ethical Standards}

Conflict of interest None.

\section{References}

1. World Health Organization (2020) Clinical management of severe acute respiratory infection (SARI) when COVID-19 disease is suspected: Interim guidance. https://www.who.int/docs/defaultsource/coronaviruse/clinical-management-of-novel-cov.pd

2. Meara JG, Leather AJ, Hagander L et al (2015) Global Surgery 2030: evidence and solutions for achieving health, welfare, and economic development. Lancet 386(9993):569-624

3. Odland ML, Whitaker J, Nepogodiev D et al (2020) Identifying, prioritizing and visually mapping barriers to injury care in Rwanda: a multi-disciplinary stakeholder exercise. World J Surg 2020:10

4. Edem IJ, Dare AJ, Byass P et al (2019) External injuries, trauma and avoidable deaths in Agincourt, South Africa: a retrospective observational and qualitative study. BMJ Open 9(6):e027576

5. Lakhoo K (2020) Global surgery. J Pediatr Surg 55(2):218-222

6. Ologunde R, Maruthappu M, Shanmugarajah K et al (2014) Surgical care in low and middle-income countries: burden and barriers. Int J Surg 12(8):858-863

Publisher's Note Springer Nature remains neutral with regard to jurisdictional claims in published maps and institutional affiliations. 